\title{
Neutron scattering studies of the structure and lattice dynamics of a solid solution of hydrogen in $\alpha$-manganese
}

\author{
V K Fedotov†, V E Antonov†, K Cornellł, G Grosse§, A I Kolesnikov†, \\ V V Sikolenko\|, V V Sumin\|, F E Wagner§ and H Wipf $\ddagger$ \\ $\dagger$ Institute of Solid State Physics, Russian Academy of Sciences, 142432 Chernogolovka, \\ Moscow District, Russia \\ $\ddagger$ Institut für Festkörperphysik, Technische Hochschule Darmstadt, Hochschulstraße 6, D-64289 \\ Darmstadt, Germany \\ $\S$ Physik-Department E 15, Technische Universität München, D-85747 Garching, Germany \\ || Frank Laboratory of Neutron Physics, Joint Institute for Nuclear Research, 141980 Dubna, \\ Moscow District, Russia \\ Received 26 January 1998
}

\begin{abstract}
A solid solution of hydrogen in $\alpha-\mathrm{Mn}, \alpha-\mathrm{MnH}_{x}$ with $x=0.073$, was prepared at $623 \mathrm{~K}$ and a hydrogen pressure of $0.85 \mathrm{GPa}$. A profile analysis of the neutron diffraction patterns measured at $225 \mathrm{~K}$ and at $300 \mathrm{~K}$ showed that hydrogen in $\alpha-\mathrm{MnH}_{0.073}$ randomly occupies the interstitial positions $12 \mathrm{e}(0,0,0.538)$ of the $\alpha$-Mn host lattice. An inelastic neutron scattering study of $\alpha-\mathrm{MnH}_{0.073}$ at $90 \mathrm{~K}$ revealed a pronounced peak at $6.4 \mathrm{meV}$ and a rather broad optical hydrogen band with peaks at 73, 105 and $123 \mathrm{meV}$. The splitting of the optical band into three different modes agrees with the low symmetry, 2, of the $12 \mathrm{e}$ hydrogen sites. A tentative interpretation is given for the peak at $6.4 \mathrm{meV}$.
\end{abstract}

\section{Introduction}

At atmospheric pressure, the equilibrium hydrogen solubility in any of the $\alpha, \beta, \gamma$ (FCC) and $\delta$ (BCC) allotropic modifications of manganese does not exceed a $\mathrm{H} / \mathrm{Mn}$ atomic ratio of $x=0.003$ [1]. Under high hydrogen pressures, manganese forms non-stoichiometric HCP [2, 3] and FCC [4, 5] hydrides with wide ranges of composition. The crystal and magnetic structures of the antiferromagnetic HCP hydrides were studied by means of neutron diffraction [6] and Mössbauer spectroscopy [7, 8], their lattice dynamics by means of inelastic neutron scattering [9]. The FCC manganese hydrides were also found to be antiferromagnets and the dependence of their Néel temperature on the hydrogen concentration was studied by means of neutron diffraction and magnetization measurements [4].

A recent high-pressure investigation of the $T-P$ phase diagram of the $\mathrm{Mn}-\mathrm{H}$ system has shown [10] that besides the HCP and FCC hydrides, rather concentrated primary solid solutions of hydrogen in the $\alpha$ and $\beta$ modifications of manganese exist, which contain up to a few atomic per cent of hydrogen. The $\alpha$ solutions were studied by Mössbauer spectroscopy using 0.2 at. ${ }^{119} \mathrm{Sn}$ as a probe [11]. These experiments showed that the Néel temperature of antiferromagnetic $\alpha$-Mn increases by about $6 \mathrm{~K}$ per atomic per cent of dissolved hydrogen.

This paper presents the results of neutron diffraction (ND) and inelastic neutron scattering (INS) investigations of an $\alpha-\mathrm{MnH}_{x}$ solution with $x=0.073$. In order to reveal 
the effects of hydrogen dissolution in $\alpha$-Mn more accurately and reliably, the spectra of the $\alpha-\mathrm{MnH}_{0.073}$ sample were compared with those of a sample of pure $\alpha-\mathrm{Mn}$ measured under the same conditions.

\section{Experimental details}

Samples of $8.45 \mathrm{~g}$ of $\alpha-\mathrm{Mn}$ and $\alpha-\mathrm{MnH}_{0.073}$ were made of powdered manganese metal of $99.99 \mathrm{wt} \%$ purity and a grain size of 0.05 to $0.1 \mathrm{~mm}$. To produce the powder, the initial plates of electrolytic manganese of about $1 \mathrm{~mm}$ thickness were machined to remove the oxidized surface layer and then crushed in an agate mortar and screened. The $\alpha-\mathrm{MnH}_{0.073}$ solid solution was prepared by exposing the powder to a hydrogen pressure of $0.85 \mathrm{GPa}$ at $623 \mathrm{~K}$ for $4 \mathrm{~h}$ with subsequent rapid cooling to ambient temperature; the method is described elsewhere [12]. In order to achieve the maximum hydrogen concentration in the $\alpha$ solid solution, the conditions of hydrogenation were chosen close to those of the transformation of the $\alpha$ solid solution to the HCP hydride [13]. The hydrogen content $x=0.073(1)$ of the $\alpha-\mathrm{MnH}_{x}$ sample obtained was determined by hot extraction into a calibrated volume at temperatures up to $800 \mathrm{~K}$ using a sample of about $20 \mathrm{mg}$. No release of hydrogen from the $\alpha-\mathrm{MnH}_{0.073}$ sample was observed at room temperature in the course of a month.

The ND experiments were performed with the D1B diffractometer at the ILL in Grenoble, with a PG(002) monochromator and neutrons of wavelength $\lambda=2.5235 \AA$, and with the high-resolution Fourier diffractometer HRFD at the pulsed reactor IBR-2 at the JINR in Dubna. The measurements with the D1B diffractometer were carried out at $225 \mathrm{~K}$, those with the HRFD at $300 \mathrm{~K}$. For each instrument, the background was determined in a separate empty-can measurement and subtracted from the measured diffraction spectra of the $\alpha-\mathrm{Mn}$ and $\alpha-\mathrm{MnH}_{0.073}$ samples. The resulting spectra were analysed using the Rietveld profile refinement technique implemented in the computer programs for the D1B [14] and the HRFD diffractometer [15].

The INS measurements were carried out at $90 \mathrm{~K}$ with the inverted-geometry time-offlight spectrometer KDSOG-M at the JINR in Dubna [16] with a Be filter and a Zn analyser and an energy of recorded neutrons of $4.8 \mathrm{meV}$. The INS spectrum of hydrogen in $\alpha$ $\mathrm{MnH}_{0.073}$ was obtained as the difference between the experimental spectra of $\alpha-\mathrm{MnH}_{0.073}$ and of $\alpha$-Mn measured under identical conditions.

An extrapolation of the Mössbauer data of reference [11] to the hydrogen content of the sample studied in this work shows that $\alpha-\mathrm{MnH}_{0.073}$ should be antiferromagnetically ordered below a Néel temperature of about $140 \mathrm{~K}$. The Néel temperature of pure $\alpha$-Mn is about $95 \mathrm{~K}$. Both $\alpha-\mathrm{MnH}_{0.073}$ and $\alpha$-Mn samples were thus studied by means of ND in the paramagnetic state and by means of INS in the antiferromagnetic state.

\section{Neutron diffraction}

The body-centred cubic unit cell of the $\alpha$-Mn structure, space group $I \overline{4} 3 m$, contains 58 atoms [17]. The $\left(\frac{1}{2}, \frac{1}{2}, \frac{1}{2}\right)$ translation in this structure links two identical sub-units of 29 atoms positioned on the four crystallographically inequivalent sites $2 \mathrm{a}, 8 \mathrm{c}, 24 \mathrm{~g}_{1}$ and $24 \mathrm{~g}_{2}$. The latter three have positional parameters which might vary on hydrogen uptake. In the case of $\alpha-\mathrm{MnH}_{0.073}$ the positional parameters of $\mathrm{Mn}$ at these sites were therefore determined together with the parameters for the hydrogen sites.

The type of the position, its occupancy and the thermal factor of hydrogen in the $\alpha$ $\mathrm{MnH}_{0.073}$ solid solution were first roughly estimated from a ND spectrum in the range 
$2.6>d_{h k \ell}>1.5 \AA$ of lattice plane distances $d_{h k \ell}$. This spectrum was measured with good statistics and low background with the D1B diffractometer. Because of the large thermal factor of hydrogen, the relative contribution of hydrogen atoms to the scattering intensity of $\alpha-\mathrm{MnH}_{0.073}$ decreases with decreasing $d_{h k \ell}$. Therefore the values of the positional parameters and the thermal factors of the manganese atoms were then refined using the $1.5>d_{h k \ell}>0.7 \AA$ part of a ND spectrum measured with the high-resolution HRFD diffractometer. The final refinement was performed by an iterative approximation procedure combining profile analyses of the D1B spectrum with $d$-values of 2.9 to $1.4 \AA$ and of the HRFD spectrum divided into three sections with $d$-values of 2.2 to $1.1,1.1$ to 0.9 and 0.9 to $0.7 \AA$.

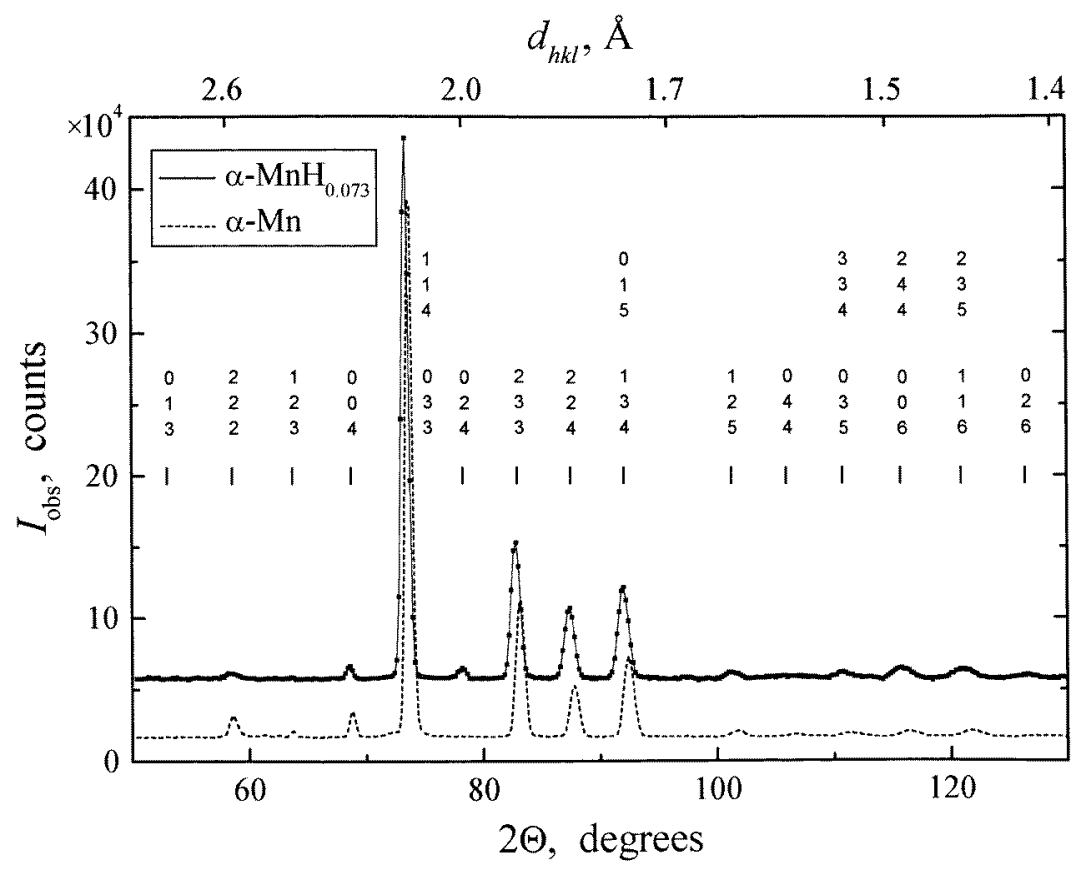

Figure 1. Experimental powder neutron diffraction patterns of $\alpha-\mathrm{MnH}_{0.073}$ (dots connected by a solid line) and $\alpha$-Mn (dashed line) measured at $225 \mathrm{~K}$ for $5 \mathrm{~h}$ with the D1B diffractometer at a wavelength $\lambda=2.5235 \AA$. The vertical bars in the upper part of the figure indicate the peak positions for $\alpha-\mathrm{MnH}_{0.073}$.

The ND spectra obtained and the results of their profile analysis are shown in figures 1-3. For the sake of clarity, the smooth incoherent component was subtracted from the spectra in figures 1 and 3 and the spectra in figure 1 were shifted with respect to each other along the intensity axis.

As is seen from figures 1 and 3, the dissolution of hydrogen in $\alpha$-Mn causes a displacement of the diffraction lines towards larger $d_{h k \ell}$ due to the hydrogen-induced lattice expansion. The relative intensities of the diffraction lines also change, but no additional lines appear on hydrogenation. In particular, the (024) line is present in both spectra in figure 1, though in the spectrum of $\alpha-\mathrm{Mn}$ its intensity is very low. One can therefore conclude that the crystal structure of the $\alpha-\mathrm{MnH}_{0.073}$ solid solution belongs to the same space group, $I \overline{4} 3 m$, as the structure of $\alpha-\mathrm{Mn}$.

Hydrogen in transition metals was found to occupy only interstitial sites which allow 


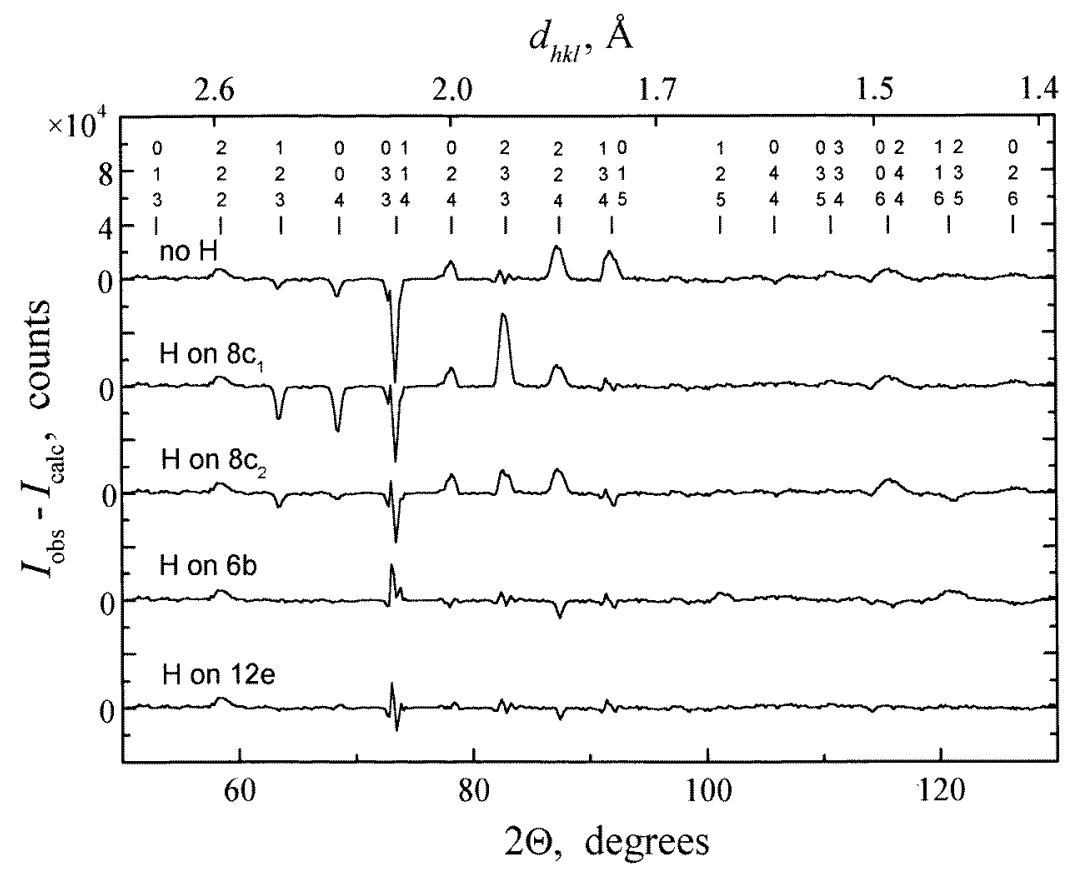

Figure 2. Differences between the experimental neutron diffraction spectrum of $\alpha-\mathrm{MnH}_{0.073}$ shown in figure 1 and the profiles calculated for different conceivable $\alpha-\mathrm{MnH}_{x}$ structures. The results of profile calculations without hydrogen (the difference spectrum labelled 'no H') and with $x=0.07$ of hydrogen randomly distributed over the interstitial positions $8 \mathrm{c}_{1}(0.109,0.109,0.109), 8 \mathrm{c}_{2}(0.206,0.206,0.206), 6 \mathrm{~b}(0,0,0.5)$ and $12 \mathrm{e}(0,0,0.538)$, respectively, are shown. All of the profiles were calculated with the parameters of the Mn atoms given in table 1 for $\alpha-\mathrm{MnH}_{0.073}$.

Table 1. Positional parameters $(x, y, z)$, thermal factors $(B)$ and site occupancies $(\omega)$ for $\alpha-\mathrm{Mn}$ and for the solid hydrogen solution $\alpha-\mathrm{MnH}_{0.073}$ according to a Rietveld profile refinement of the neutron diffraction data collected with the HRFD (JINR) diffractometer. $R_{p}$ and $R_{e x}$ are the obtained and expected profile factors. Cubic structure, space group $I \overline{4} 3 m$ (No 217), $T=300 \mathrm{~K}$.

\begin{tabular}{lllllllll}
\hline \multirow{2}{*}{ Phase } & Atom & Site & $\begin{array}{l}\text { Site } \\
\text { symmetry }\end{array}$ & $x$ & $y$ & $z$ & $B\left(\AA^{2}\right)$ & $\omega$ \\
\hline$\alpha-\mathrm{Mn}$ & $\mathrm{Mn}$ & $2 \mathrm{a}$ & $\overline{4} 3 m$ & 0 & 0 & 0 & 0.72 & 1.00 \\
$a=8.9138(3) \AA$ & $\mathrm{Mn}$ & $8 \mathrm{c}$ & $3 m$ & $0.315(2)$ & $0.315(2)$ & $0.315(2)$ & 0.45 & 1.00 \\
$R_{p} / R_{e x}=5.0 / 3.1$ & $\mathrm{Mn}$ & $24 \mathrm{~g}_{1}$ & $m$ & $0.354(2)$ & $0.354(2)$ & $0.033(2)$ & 0.48 & 1.00 \\
& $\mathrm{Mn}$ & $24 \mathrm{~g}_{2}$ & $m$ & $0.090(2)$ & $0.090(2)$ & $0.280(2)$ & 0.44 & 1.00 \\
\hline$\alpha-\mathrm{MnH}_{0.073}$ & $\mathrm{Mn}$ & $2 \mathrm{a}$ & $\overline{4} 3 m$ & 0 & 0 & 0 & 0.74 & 1.00 \\
$a=8.9403(3) \AA$ & $\mathrm{Mn}$ & $8 \mathrm{c}$ & $3 m$ & $0.319(2)$ & $0.319(2)$ & $0.319(2)$ & 0.45 & 1.00 \\
$R_{p} / R_{e x}=5.2 / 4.0$ & $\mathrm{Mn}$ & $24 \mathrm{~g}_{1}$ & $m$ & $0.355(1)$ & $0.355(1)$ & $0.034(1)$ & 0.61 & 1.00 \\
& $\mathrm{Mn}$ & $24 \mathrm{~g}_{2}$ & $m$ & $0.091(1)$ & $0.091(1)$ & $0.281(1)$ & 0.45 & 1.00 \\
& $\mathrm{H}$ & $12 \mathrm{e}$ & 2 & 0 & 0 & $0.538(2)$ & 1.57 & 0.34 \\
\hline
\end{tabular}

the accommodation of atoms with an effective radius of $0.56-0.60 \AA$ [18], corresponding to a distance of $R(\mathrm{H}-\mathrm{Me}) \approx 1.8-2.0 \AA$ between the hydrogen atom and the nearest metal atoms. In HCP hydrides of manganese, for instance, $R(\mathrm{H}-\mathrm{Mn}) \approx 1.9 \AA$ is observed [6]. 
Thus, in the case of $\alpha-\mathrm{MnH}_{0.073}$ only positions of the space group $I \overline{4} 3 m$ located inside interstices of the proper size had to be analysed for hydrogen occupancy.

In the crystal structure of $\alpha-\mathrm{Mn}$, there are only two types of interstice with volumes large enough to satisfy the restrictions given in [18]. These are interstices around $8 \mathrm{c}(x, x, x)$ positions with $x=0.109$ and $x=0.206$, which are the centres of tetrahedra of manganese atoms at $24 \mathrm{~g}_{2}$ and $2 \mathrm{a}$ sites and at $24 \mathrm{~g}_{1}$ and $8 \mathrm{c}$ sites, respectively, and have distances $R(8 \mathrm{c}-\mathrm{Mn})$ of about $1.7 \AA$. The second type is an interstice around the $6 \mathrm{~b}(0,0,0.5)$ position, which is the common centre of two tetrahedra of Mn atoms at $24 \mathrm{~g}_{1}$ sites with $R(6 \mathrm{~b}-\mathrm{Mn})=1.858 \AA$ and at $24 \mathrm{~g}_{2}$ sites with $R(6 \mathrm{~b}-\mathrm{Mn})=2.272 \AA$.

At the beginning of the structure refinement procedure, the hydrogen atoms were tentatively placed at the above positions of the space group $I \overline{4} 3 \mathrm{~m}$ at the centres of the interstices. The differences between the measured spectrum and spectra calculated under these assumptions are shown in figure 2 .

Figure 2 also shows a difference spectrum calculated on the assumption that none of the interstitial sites is occupied by hydrogen. This curve, labelled 'no H', shows the effect of dissolved hydrogen on the line intensities in the ND spectrum of $\alpha-\mathrm{MnH}_{0.073}$. The only exception is a broad peak near the (222) line which is observed also in the difference spectrum of the initial $\alpha$-Mn sample. We attribute these peaks in the $\alpha$-Mn and $\alpha-\mathrm{MnH}_{0.073}$ spectra to a fine-grain surface impurity and excluded them from the calculation scheme. All other peaks in the 'no H' difference spectrum are due to neutron scattering by hydrogen and have to be described by the profile modelling.

A comparison with the 'no H' curve shows that the models with hydrogen atoms at the $8 c_{1}(0.109,0.109,0.109)$ and the $8 c_{2}(0.206,0.206,0.206)$ sites are inadmissible. Up to $2 \Theta=100^{\circ}$, corresponding to $d_{h k \ell}>1.65 \AA$, the model with hydrogen at the $6 \mathrm{~b}(0,0,0.5)$ site agrees with experiment rather well. At $d_{h k \ell}<1.65 \AA$, however, the calculated intensities differ noticeably from the observed ones in the spectra of $\alpha-\mathrm{MnH}_{0.073}$ measured with both the D1B and HRFD diffractometers, and this difference cannot be eliminated by varying the positional parameters of the $\mathrm{Mn}$ atoms. In addition, the profile refinement gave a value of $x \approx 0.05$ for the hydrogen content of the sample, instead of $x=0.073$ as determined by hot extraction. These discrepancies lead to the conclusion that the actual hydrogen position in $\alpha-\mathrm{MnH}_{0.073}$ is close to but is not exactly a $6 \mathrm{~b}$ position.

A model with hydrogen displaced from the $6 \mathrm{~b}(0,0,0.5)$ site to a $12 \mathrm{e}(0,0, z)$ site with a variable parameter $z$ close to 0.5 gave a significantly better agreement between the calculation and experiment. With $z=0.538$ the ratio $R_{p} / R_{e x}$ of obtained and expected profile factors fell by approximately $20 \%$ and the refined value of the hydrogen content increased to $x \approx 0.07$, which agrees with the result of the hot extraction. The quality of the profile fitting is illustrated in figure 2 by the difference spectrum labelled ' $\mathrm{H}$ on $12 \mathrm{e}$ ' and in figure 3 by the agreement between the calculated profile shown as a solid curve and the experimental points.

The final refined values of the structure parameters of $\alpha-\mathrm{MnH}_{0.073}$ are presented in table 1 together with those of $\alpha-\mathrm{Mn}$ determined in a similar manner. In the course of refinement of the $\alpha-\mathrm{MnH}_{0.073}$ and $\alpha$-Mn structures, the parameters $\omega$ of the site occupancy for $\mathrm{Mn}$ atoms were fixed to unity, and the other structure parameters listed in table 1 were subject to fitting. Within the experimental error the coordinates obtained for the Mn atoms in the unit cell of $\alpha$-Mn coincide with the values given in [19] and do not change on hydrogen uptake. The increase in the atomic volume, $V_{a}$, of $\alpha$-Mn caused by the dissolved hydrogen corresponds to a value of $\partial V_{a} / \partial x=1.50(4) \AA^{3} / \mathrm{H}$ atom which lies near the lower limit of the interval 1.5-3 $\AA^{3} / \mathrm{H}$ atom characteristic of hydrides of transition metals [12, 20].

It is worth noting that on physical grounds the suggested displacement of hydrogen from 

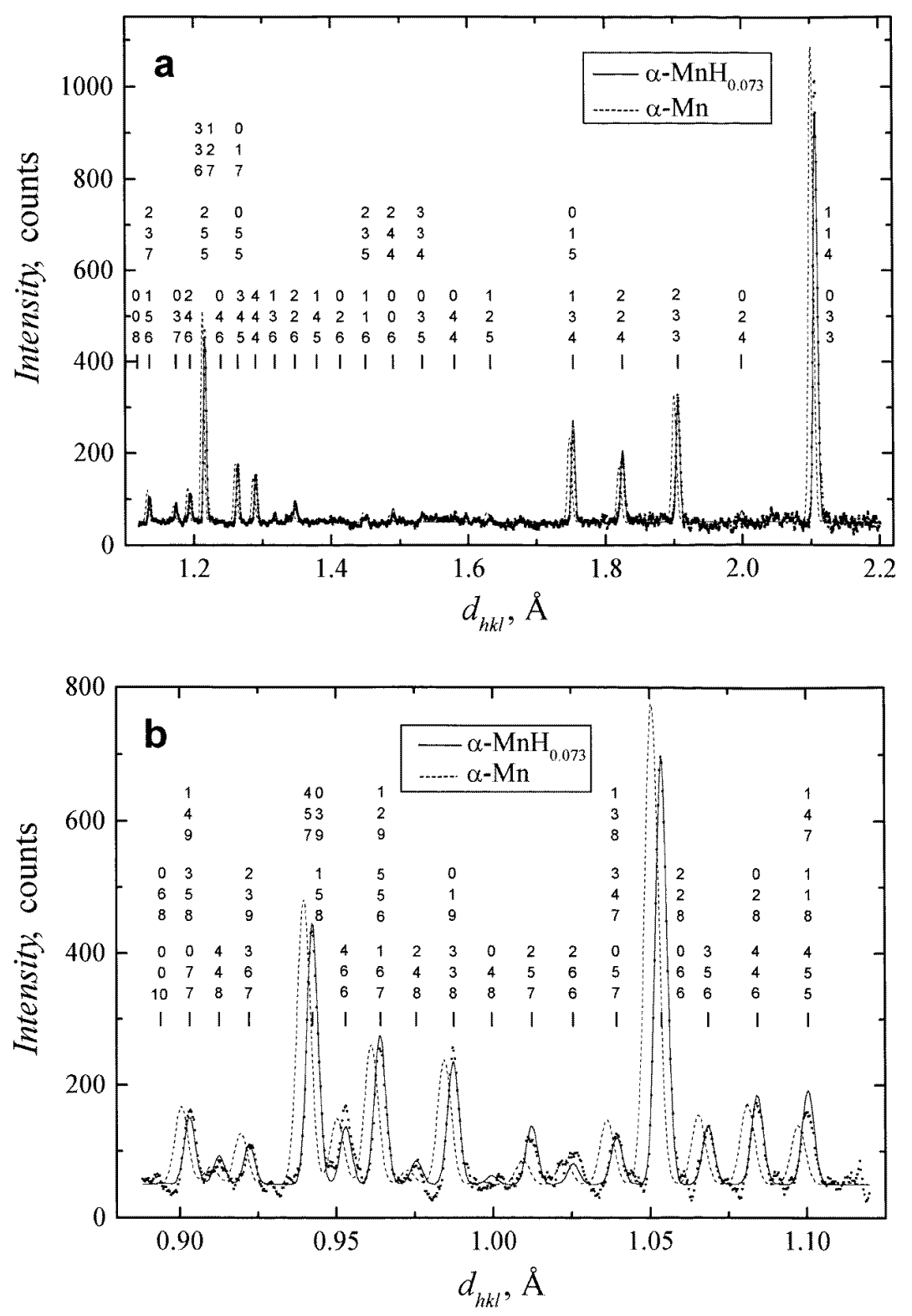

Figure 3. Experimental intensities for $\alpha-\mathrm{MnH}_{0.073}$ (dots) and profiles resulting from the Rietveld analysis of the powder neutron diffraction patterns of $\alpha$ - Mn (dashed line) and $\alpha-\mathrm{MnH}_{0.073}$ (solid line) measured at $300 \mathrm{~K}$ for $10 \mathrm{~h}$ with the time-of-flight diffractometer HRFD. The vertical bars in the upper part of the figure indicate the peak positions for $\alpha-\mathrm{MnH}_{0.073}$. Three different ranges of lattice plane distances, $d_{h k \ell}$, are shown in separate plots.

the $6 \mathrm{~b}$ to a $12 \mathrm{e}$ position is the most likely one. As is shown in figure 4(a), the $6 \mathrm{~b}$ site lies on a $\overline{4}$ axis and the interstice around it is formed by two tetrahedra of Mn atoms rotated with respect to each other by an angle of $90^{\circ}$ about this axis. The $\overline{4}$ axis is an essential element of the $6 \mathrm{~b}$ site symmetry $\overline{4} 2 \mathrm{~m}$, so one could expect the displacement of hydrogen 


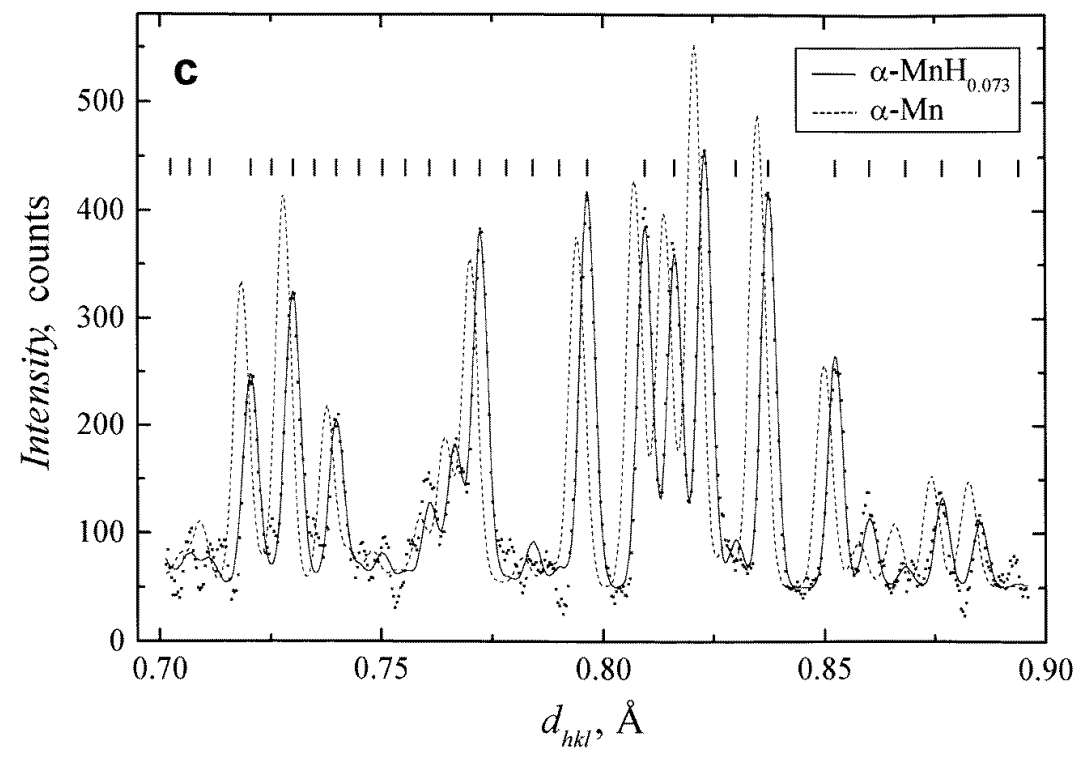

Figure 3. (Continued)
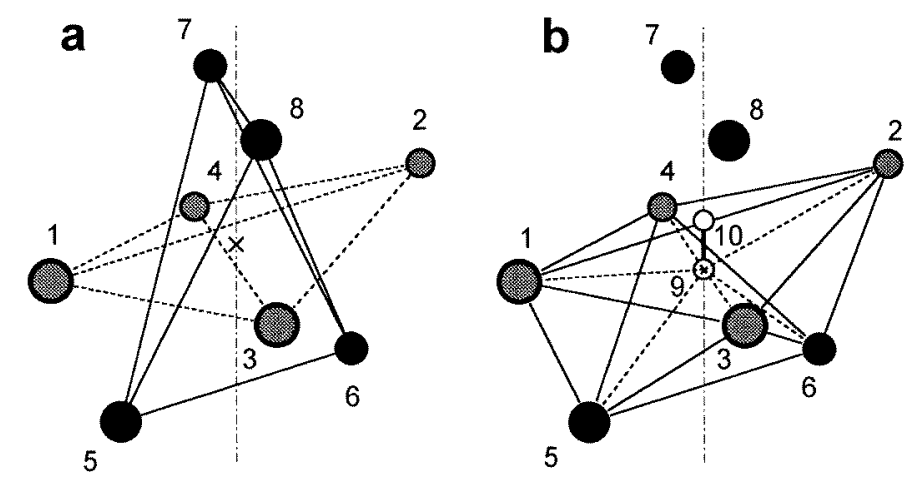

(2)- Mn on $24 g_{1} \bigcirc-$ Mn on $24 g_{2} \quad x-6 b$ position $\mathrm{O}-\mathrm{H}$ on $12 \mathrm{e}$

Figure 4. The position $6 \mathrm{~b}$ (the cross, (a)), and the two adjacent positions $12 \mathrm{e}$ partly occupied one at a time by hydrogen (open circles 9 and 10, (b)) and their nearest neighbourhood of two tetrahedra formed by manganese atoms at positions $24 \mathrm{~g}_{1}$ (shaded circles 1 to 4 ) and at positions $24 \mathrm{~g}_{2}$ (solid circles 5 to 8 ) in the crystal structure of $\alpha-\mathrm{MnH}_{0.073}$. The vertical chain lines show the $\overline{4}$ axis which is a symmetry element of the space group $I \overline{4} 3 m$ of $\alpha-\mathrm{Mn}$ and $\alpha-\mathrm{MnH}_{0.073}$ and also one of the $6 \mathrm{~b}$ site symmetry $\overline{4} 2 m$.

to occur along this very axis. Any position on this axis different from the $6 \mathrm{~b}$ position is called a $12 \mathrm{e}$ position.

A displacement of hydrogen from the $6 \mathrm{~b}$ position along the $\overline{4}$ axis appears preferable because it leads to a decrease in the distance $R(6 \mathrm{~b}-\mathrm{Mn})=2.272 \AA$ to the nearest Mn atoms on the $24 \mathrm{~g}_{2}$ sites, which is beyond the range of $R(\mathrm{H}-\mathrm{Me}) \approx 1.8-2.0 \AA$ characteristic of most Me-H phases [18]. The distance $R(6 \mathrm{~b}-\mathrm{Mn})=1.858 \AA$ to the nearest $\mathrm{Mn}$ atoms at $24 \mathrm{~g}_{1}$ sites, which is close to the lower limit of this range, is decreased only slightly. Any 


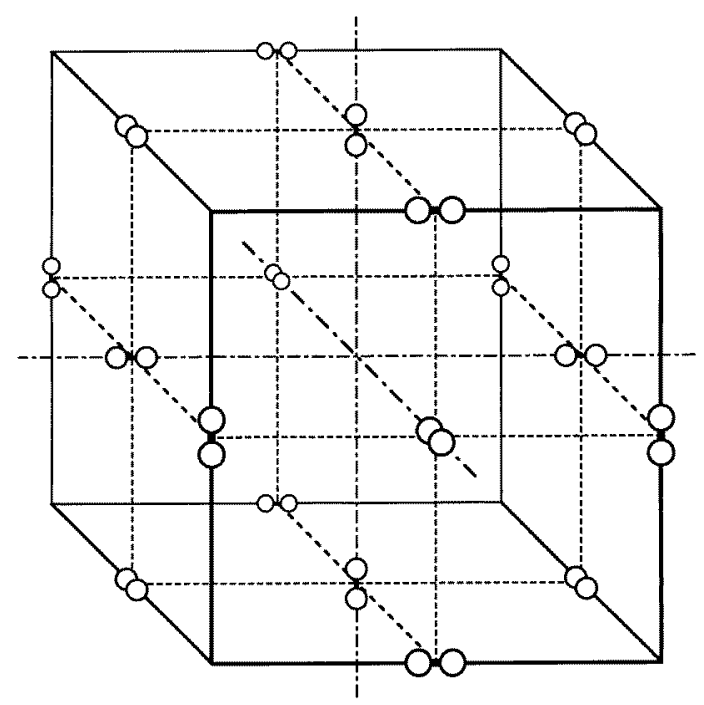

Figure 5. The arrangement of dumb-bells of $12 \mathrm{e}$ positions partly filled with hydrogen in the unit cell of $\alpha-\mathrm{MnH}_{0.073}$. Each dumb-bell can accommodate only one hydrogen atom because of the 'blocking effect' [18]. The dumb-bells on edges and at face centres are crystallographically equivalent and are linked by the BCC translation $\left(\frac{1}{2}, \frac{1}{2}, \frac{1}{2}\right)$.

Table 2. Positional parameters $(x, y, z)$ of the atoms in $\alpha-\mathrm{MnH}_{0.073}$ shown in figure 4(b) and the distances $R(9-\mathrm{i})$ between the $\mathrm{H}(9)$ atom and other atoms. The listed values of $R(9-\mathrm{i})$ are for $12 \mathrm{e}$ positions lying on the $z$-axis.

\begin{tabular}{lrrll}
\hline Atom(i) & \multicolumn{1}{c}{$x(\mathrm{i})$} & \multicolumn{1}{c}{$y(\mathrm{i})$} & $z(\mathrm{i})$ & $R(9-\mathrm{i})(\AA)$ \\
\hline $\mathrm{Mn}(1)$ & -0.145 & -0.145 & 0.534 & 1.943 \\
$\mathrm{Mn}(2)$ & 0.145 & 0.145 & 0.534 & 1.943 \\
$\mathrm{Mn}(3)$ & -0.145 & 0.145 & 0.466 & 1.834 \\
$\mathrm{Mn}(4)$ & 0.145 & -0.145 & 0.466 & 1.834 \\
$\mathrm{Mn}(5)$ & -0.091 & -0.091 & 0.281 & 1.986 \\
$\mathrm{Mn}(6)$ & 0.091 & 0.091 & 0.281 & 1.986 \\
$\mathrm{Mn}(7)$ & -0.091 & 0.091 & 0.719 & 2.570 \\
$\mathrm{Mn}(8)$ & 0.091 & -0.091 & 0.719 & 2.570 \\
$\mathrm{H}(9)$ & 0 & 0 & 0.462 & 0 \\
$\mathrm{H}(10)$ & \multicolumn{1}{c}{0} & 0 & 0.538 & 0.679 \\
\hline
\end{tabular}

displacement off the $\overline{4}$ axis would effectively decrease the latter distances.

In the cubic unit cell of $\alpha-\mathrm{MnH}_{0.073}$, the $6 \mathrm{~b}$ positions are located at the mid-points of the edges and at the centres of the faces. The 12e positions form dumb-bells centred on the $6 \mathrm{~b}$ positions and aligned along the edges and perpendicular to the faces, respectively. This is illustrated in figure 5. As is shown in figure 4(b), the displacement of the $12 \mathrm{e}$ positions (9 and 10) with respect to the $6 \mathrm{~b}$ positions is such that they are shifted from the centre of the volume close to the mid-points of the edges of tetrahedra formed by manganese atoms ( 1 to 4 ) at positions $24 \mathrm{~g}_{1}$. As is seen from the interatomic distances given in table 2 , the nearest neighbours of the position 12e (9) are four Mn atoms ( 1 to 4 ) at positions $24 \mathrm{~g}_{1}$ and two $\mathrm{Mn}$ atoms $(5,6)$ at positions $24 \mathrm{~g}_{2}$. These $\mathrm{Mn}$ atoms form a distorted octahedron (1 to 6) around the position 12e (9). The more distant Mn atoms $(7,8)$ together with the $\mathrm{Mn}$ 
atoms ( 1 to 4 ) form a similar octahedron around the other $12 \mathrm{e}$ position (10).

The $12 \mathrm{e}(0,0,0.538)$ position of hydrogen satisfies the empirical rule of reference [18] better than the $6 \mathrm{~b}(0,0,0.5)$ position. As is seen from table 2 , the values of the $R(12 \mathrm{e}-\mathrm{Mn})$ distances between a hydrogen atom and the six nearest $\mathrm{Mn}$ atoms in $\alpha-\mathrm{MnH}_{0.073}$ lie well within the limits of $R(\mathrm{H}-\mathrm{Me}) \approx 1.8-2.0 \AA$ characteristic of the phases formed by hydrogen with transition metals [18]. Moreover, the $R(12 \mathrm{e}-\mathrm{Mn})$ distances differ from each other much less than the $R(6 \mathrm{~b}-\mathrm{Mn})$ distances. Thus, despite its lower symmetry, the interstice enclosing the $12 \mathrm{e}$ site is more 'spherical' than that enclosing the $6 \mathrm{~b}$ site.

As also indicated in table 2 , the distance $R(9-10)$ between the two $12 \mathrm{e}$ positions within a dumb-bell is about $0.68 \AA$. Because of this small distance, these positions cannot be occupied by hydrogen at the same time due to the 'blocking effect' [18] requiring that the distance between hydrogen atoms in a metal should not be much less than $2 \AA$. Occupancy of half of the $12 \mathrm{e}$ sites corresponds to a H/Mn atomic ratio of $x=6 / 58 \approx 0.103$. In the $\alpha-\mathrm{MnH}_{x}$ sample studied, with $x=0.073$, therefore about $70 \%$ of the accessible interstices are occupied.

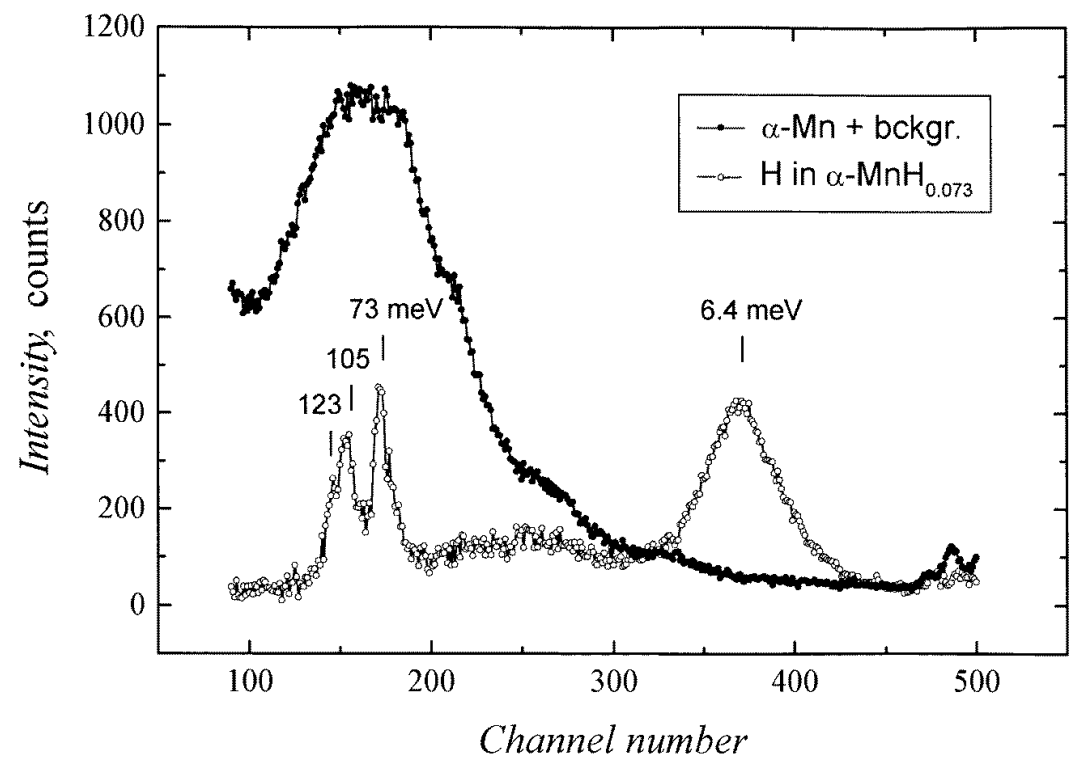

Figure 6. The experimental INS spectrum of $\alpha-\mathrm{Mn}$ (solid circles) and the difference between the INS spectra of $\alpha-\mathrm{MnH}_{0.073}$ and $\alpha$-Mn representing the spectrum of hydrogen dissolved in $\alpha-\mathrm{MnH}_{0.073}$ (open circles). The spectra of both $\alpha-\mathrm{Mn}$ and $\alpha-\mathrm{MnH}_{0.073}$ were measured with the time-of-flight spectrometer KDSOG-M at $90 \mathrm{~K}$ for $30 \mathrm{~h}$ each.

\section{Inelastic neutron scattering}

The KDSOG-M time-of-flight spectrometer used in this work has a high neutron flux at the sample position and provides a medium resolution $\Delta \mathcal{E} / \mathcal{E}=4-6 \%$ for energy transfers between 1 and $60 \mathrm{meV}$ and $\Delta \mathcal{E} / \mathcal{E}=6-10 \%$ at higher energy transfers between 60 and $200 \mathrm{meV}$. To reduce the contribution from multi-phonon scattering, the INS spectra of $\alpha-\mathrm{Mn}$ and $\alpha-\mathrm{MnH}_{0.073}$ were measured at a temperature of $90 \mathrm{~K}$. The samples weighed $8.45 \mathrm{~g}$ each, and the data were collected for $30 \mathrm{~h}$ simultaneously at four scattering angles of $2 \Theta=80$, 
100,120 and $140^{\circ}$. The four spectra obtained were then added to ensure the validity of the incoherent approximation used in the evaluation.

To illustrate the magnitude of the effect of hydrogen on the INS spectrum of $\alpha-\mathrm{Mn}$, the experimental spectrum of the $\alpha$-Mn sample and the difference between the spectra of the $\alpha-\mathrm{MnH}_{0.073}$ and $\alpha-\mathrm{Mn}$ samples are shown in figure 6. The huge dome around channel 160 in the $\alpha$-Mn spectrum originates from neutron scattering by the sample and its environment, including the aluminium sample holder, cryostat, etc, and reflects the spectral density of the incident neutron flux. The difference spectrum is expected to be close to the INS spectrum of hydrogen in $\alpha-\mathrm{MnH}_{0.073}$, because the small amount of hydrogen dissolved in $\alpha-\mathrm{Mn}$ can hardly change the vibrational spectrum of the host-lattice metal atoms significantly. This can be inferred, for example, from a comparison with the changes in the spectrum of metal vibrations due to formation of hydrides in the $\mathrm{Ni}-\mathrm{H}$ and $\mathrm{Pd}-\mathrm{H}$ systems [21], where the hydrides have the same structure type as the pure metals.

As is seen from figure 6, the INS spectrum of hydrogen in $\alpha-\mathrm{MnH}_{0.073}$ exhibits peaks in the region of energy transfer from 50 to $150 \mathrm{meV}$ characteristic of optical vibrations of hydrogen in metal-hydrogen systems, and also a pronounced peak at $6.4 \mathrm{meV}$.

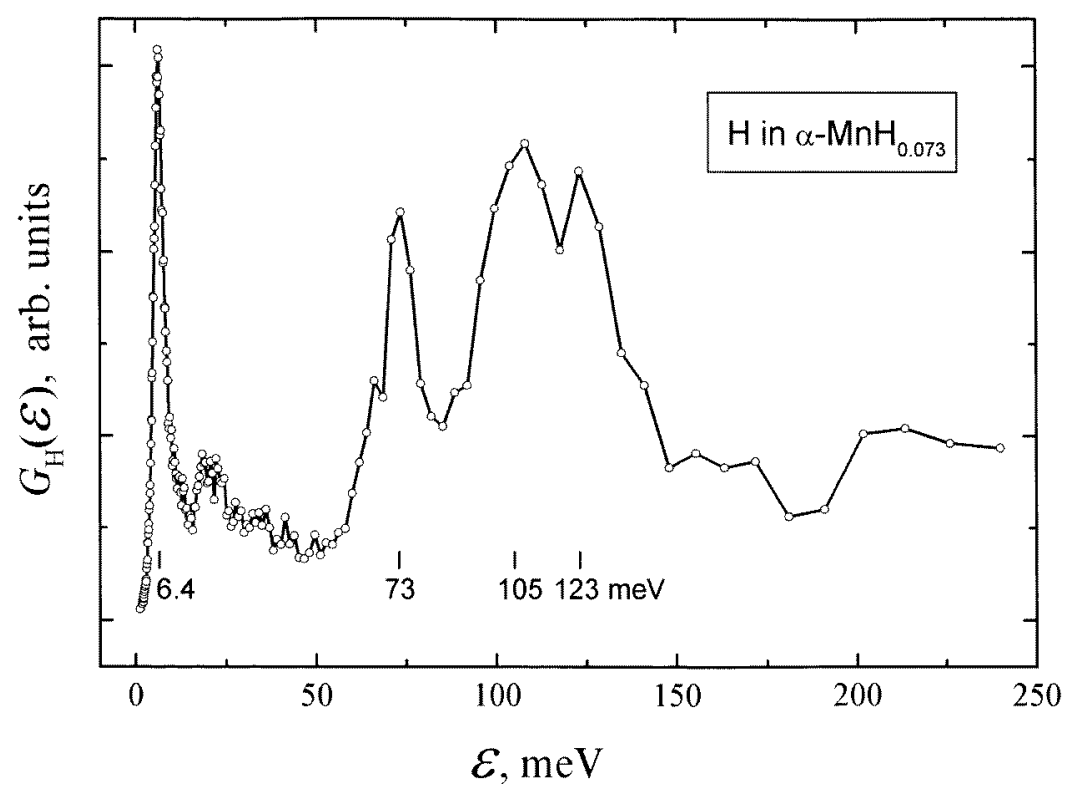

Figure 7. The generalized vibrational density of states, $G_{\mathrm{H}}(\mathcal{E})$, of hydrogen in $\alpha-\mathrm{MnH}_{0.073}$ at $90 \mathrm{~K}$ obtained from the difference between the experimental INS spectra of $\alpha-\mathrm{MnH}_{0.073}$ and of $\alpha$-Mn shown in figure 6.

Figure 7 shows this spectrum transformed to the generalized vibrational density of states $G_{\mathrm{H}}(\mathcal{E})$, the standard data representation at the KDSOG-M spectrometer. The $G_{\mathrm{H}}(\mathcal{E})$ spectrum can be divided into three regions: the region of the lattice band modes at $\mathcal{E}<50 \mathrm{meV}$ with a sharp peak at $6.4 \mathrm{meV}$, the region of the optical band of hydrogen with three rather well resolved peaks at 73,105 and $123 \mathrm{meV}$, and a region at higher energies, in which multi-phonon processes become dominant.

The splitting of the optical band of hydrogen in $\alpha-\mathrm{MnH}_{0.073}$ into three different modes agrees with the low site symmetry, 2 , of the $12 \mathrm{e}$ position. The peak at $73 \mathrm{meV}$ presumably 
corresponds to a low-energy mode of hydrogen vibrations along the $\overline{4}$ axis, because the nearest-neighbour Mn atoms are most distant and symmetrically positioned relative to this line (figure 4 and table 2). The peaks at 105 and $123 \mathrm{meV}$ can be attributed to vibrations perpendicular to this line. Note that for hydrogen occupying the $6 \mathrm{~b}$ position with a higher site symmetry, $\overline{4} 2 \mathrm{~m}$, the high-energy local mode of hydrogen vibrations perpendicular to the $\overline{4}$ axis would be degenerate.

The peak at $6.4 \mathrm{meV}$ may tentatively be explained by hydrogen tunnelling between adjacent $12 \mathrm{e}$ positions. With an energy $\mathcal{E}_{0}=73 \mathrm{meV}$ of the local vibrations of hydrogen atoms along the line connecting two sites at a distance of $0.68 \AA$ from each other, a simple model [22] allows a rough estimate of the splitting $\Delta_{0}$ of the hydrogen vibrational ground state due to tunnelling and gives

$$
\Delta_{0}=\frac{1}{2} \mathcal{E}_{0} \exp \left(-M_{\mathrm{H}} \mathcal{E}_{0} l^{2} / \hbar^{2}\right) \approx 5 \mathrm{meV}
$$

where $M_{\mathrm{H}}$ is the mass of hydrogen atom and $2 l$ is the distance between the two sites. The estimated value of $\Delta_{0} \approx 5 \mathrm{meV}$ agrees quite well with the energy of $6.4 \mathrm{meV}$ of the peak in the INS spectrum of $\alpha-\mathrm{MnH}_{0.073}$.

Further investigations of the temperature, neutron momentum transfer and isotope dependencies of the peak position and intensity are necessary, however, for an unambiguous interpretation. Such work is in progress.

\section{Summary}

Under high hydrogen pressures, the equilibrium solubility of hydrogen in the lowtemperature $\alpha$ modification of manganese increases to up to a few atomic per cent. In the present work, an $\alpha-\mathrm{MnH}_{0.073}$ sample synthesized at $623 \mathrm{~K}$ and at a hydrogen pressure of $0.85 \mathrm{GPa}$ was studied at ambient pressure by means of neutron diffraction and inelastic neutron scattering.

The profile analysis of the neutron diffraction patterns of $\alpha-\mathrm{MnH}_{0.073}$ measured at $225 \mathrm{~K}$ and at $300 \mathrm{~K}$ showed that hydrogen randomly occupies interstitial positions of the 12e type inside distorted octahedra of manganese atoms. The dissolution of hydrogen results in an increase in the lattice parameter of $\alpha-\mathrm{Mn}$, but the coordinates of the $\mathrm{Mn}$ atoms in the unit cell remain unchanged. The $12 \mathrm{e}$ sites occupied by hydrogen form dumb-bells positioned at the centres of the edges and faces of the unit cell of $\alpha-\mathrm{Mn}$. Because of the small distance of about $0.68 \AA$ between the $12 \mathrm{e}$ sites in a dumb-bell, these sites cannot be occupied by hydrogen simultaneously. Occupancy of half of the $12 \mathrm{e}$ sites corresponds to an H/Mn atomic ratio of $x=6 / 58 \approx 0.103$. In the $\alpha-\mathrm{MnH}_{0.073}$ sample studied, hydrogen therefore filled about $70 \%$ of the accessible interstices.

The inelastic neutron scattering study of $\alpha-\mathrm{MnH}_{0.073}$ at $90 \mathrm{~K}$ revealed a strong peak at $6.4 \mathrm{meV}$ in the region of the lattice band modes and three peaks at 73, 105 and $123 \mathrm{meV}$ in the range of the optical vibrations of hydrogen. The splitting of the band of optical hydrogen vibrations into three different modes agrees with the low site symmetry, 2, of the $12 \mathrm{e}$ position. The peak at $73 \mathrm{meV}$ corresponds to a low-energy mode of hydrogen vibrations along the $\overline{4}$ axis of the dumb-bells of the $12 \mathrm{e}$ positions, while the peaks at 105 and $123 \mathrm{meV}$ are due to the vibrations perpendicular to this axis. The peak at $6.4 \mathrm{meV}$ is tentatively attributed to the splitting of the vibrational ground state of hydrogen due to tunnelling between the two 12e sites forming a dumb-bell. 


\section{Acknowledgments}

The assistance of Dr A W Hewat and Dr B Ouladdiaf, ILL, in conducting the neutron diffraction experiments is gratefully acknowledged. The work was supported by the Grants No 96-02-17522 and No 96-15-96806 from the Russian Foundation for Basic Research and by the NATO Linkage Grant No 921403.

\section{References}

[1] Hansen M and Anderko K 1958 Constitution of Binary Alloys (New York: McGraw-Hill) pp 784-5

[2] Krukowski M and Baranowski B 1975 Rocz. Chem. 49 1183-6

[3] Ponyatovskii E G and Belash I T 1975 Dokl. Akad. Nauk 224 607-8

[4] Fukai Y, Ishikawa H, Goto T, Susaki J, Yagi T, Soubeyroux J L and Fruchart D 1989 Z. Phys. Chem., NF 163 479-82

[5] Filipek S M, Majchrzak S, Sawaoka A B and Cernansky M 1991 High Pressure Res. 7 271-3

[6] Irodova A V, Glazkov V P, Somenkov V A, Shil'shtein S Sh, Antonov V E and Ponyatovskii E G 1987 Fiz. Tverd. Tela 29 2714-20 (Engl. Transl. 1987 Sov. Phys.-Solid State 29 1562-5)

[7] Schneider G, Baier M, Wagner F E, Antonov V E, Antonova T E, Kopilovskii Yu and Makarov E 1991 Hyperfine Interact. 69 467-70

[8] Grosse G, Baier M, Schneider G F, Antonov V E and Antonova T E 1996 Int. Conf. on the Applications of the Mössbauer Effect 1995 (Conf. Proc. 50) ed I Ortalli (Boulogne: SIF) pp 215-8

[9] Antonov V E, Belash I T, Kolesnikov A I, Mayer J, Natkaniec I, Ponyatovskii E G and Fedotov V K 1991 Fiz. Tverd. Tela 33 152-7 (Engl. Transl. 1991 Sov. Phys.-Solid State 33 87-90)

[10] Antonov V E, Antonova T E, Chirin N A, Ponyatovsky E G, Baier M and Wagner F E 1996 Scr. Mater. 34 $1331-6$

[11] Grosse G, Wagner F E, Antonov V E and Antonova T E 1998 Hyperfine Interact. C at press

[12] Ponyatovsky E G, Antonov V E and Belash I T 1984 Problems in Solid-State Physics ed A M Prokhorov and A S Prokhorov (Moscow: Mir) pp 109-72

[13] Krukowski M and Baranowski B 1976 J. Less-Common Met. 49 385-8

[14] Yang R A, Sakthivel A, Moss T S and Paiva-Santos C O 1995 DBWS 9411 User's Guide (Atlanta, GA: Georgia Institute of Technology)

[15] Zlokazov V B and Chernyshev V V 1992 J. Appl. Crystallogr. 25 447-51

[16] Belushkin A V (ed) 1991 User Guide, Neutron Experimental Facilities at JINR (Dubna: JINR) 72 pp

[17] Pearson W B 1964 A Handbook of Lattice Spacings and Structures of Metals and Alloys (Oxford: Pergamon) pp 734-5

[18] Somenkov V A and Shil'stein S Sh 1979 Z. Phys. Chem., NF 117 125-44

[19] Gazzara C P, Middleton R M, Weiss R J and Hall E O 1967 Acta Crystallogr. 22 859-62

[20] Peisl H 1978 Hydrogen in Metals I ed G Alefeld and J Völkl (Berlin: Springer) pp 53-74

[21] Kolesnikov A I, Natkaniec I, Antonov V E, Belash I T, Fedotov V K, Krawczyk J, Mayer J and Ponyatovsky E G 1991 Physica B 174 257-61

[22] Drechsler S L, Vujičić G M and Plakida N M 1984 J. Phys. F: Met. Phys. 14 L243-6 